\title{
Neuronal uptake of serum albumin is associated with neuron damage during the development of epilepsy
}

\author{
ZANHUA LIU ${ }^{*}$, JINJIE LIU ${ }^{2 *}$, SUPING WANG $^{1}$, SIBO LIU $^{3}$ and YONGBO ZHAO ${ }^{4}$ \\ ${ }^{1}$ Department of Neurology; ${ }^{2}$ No. 2 VIP Ward; ${ }^{3}$ Surgical Intensive Care Unit,
} Dalian Municipal Central Hospital Affiliated to Dalian Medical University, Dalian, Liaoning $11600 ;{ }^{4}$ Department of Neurology, Shanghai First People's Hospital Affiliated to Shanghai Jiao Tong University School of Medicine, Shanghai 200080, P.R. China

Received February 20, 2015; Accepted March 22, 2016

DOI: $10.3892 /$ etm.2016.3397

\begin{abstract}
It is well established that brain blood barrier dysfunction following the onset of seizures may lead to serum albumin extravasation into the brain. However, the effect of albumin extravasation on the development of epilepsy is yet to be fully elucidated. Previous studies have predominantly focused on the effect of albumin absorption by astrocytes; however, the present study investigated the effects of neuronal uptake of albumin in vitro and in kainic acid-induced Sprague-Dawley rat models of temporal lobe epilepsy. In the present study, electroencephalogram recordings were conducted to record seizure onset, Nissl and Evans blue staining were used to detect neuronal damage and albumin extravasation, respectively, and double immunofluorescence was used to explore neuronal absorption of albumin. Cell counting was also conducted in vitro to determine whether albumin contributes to neuronal death. The results of the present study indicated that extravasated serum albumin was absorbed by neurons, and the neurons that had absorbed albumin died and were dissolved 28 days after seizure onset in vivo. Furthermore, significant neuronal death was detected after albumin absorption in vitro in a dose- and time-dependent manner. These results suggested that albumin may be absorbed by neurons following the onset of seizures. Furthermore, the results indicated that neuronal albumin uptake may be associated with neuronal damage and death in epileptic seizures. Therefore, attenuating albumin extravasation following epileptic seizures may reduce brain damage and slow the development of epilepsy.
\end{abstract}

Correspondence to: Professor Yongbo Zhao, Department of Neurology, Shanghai First People's Hospital Affiliated to Shanghai Jiao Tong University School of Medicine, 85 Wujing Road, Shanghai 200080, P.R. China

E-mail: liujinjie4581583@126.com

*Contributed equally

Key words: epilepsy, seizure, albumin, neuron

\section{Introduction}

Epilepsy is a neurological disease that is characterized by the experiencing of more than two seizure attacks and the possibility of future recurrences (1). Previous studies have demonstrated vascular involvement in epilepsy, in particular brain blood barrier (BBB) dysfunction has been associated with the development of epilepsy $(2,3)$.

It is well established that the BBB is a specialized physical and chemical barrier, which is composed of tight junctions between endothelial cells (ECs), ECs, synapse of glial cells and basal lamina (4). It has previously been demonstrated that the structure and function of BBB may be significantly damaged following an epileptic seizure, which may facilitate vast molecular and cellular exchange between brain parenchyma and peripheral blood $(5,6)$. Among these molecules, albumin is the most abundant protein constituent of human blood plasma (7). Previous studies have indicated that serum albumin leakage into the brain parenchyma may contribute to epileptogenesis and the progress of epileptopathology (7) by lowering the threshold of spreading depolarization (8), inducing neuronal hyper-excitability and impairing neuronal function (9), which is inconsistent with the previously held view that serum albumin has a neuroprotective role $(10,11)$. Furthermore, previous studies have reported that extravasated albumin is capable of influencing neuronal excitability through astrocytes $(12,13)$. After transient exposure to the brain, albumin accumulates in astrocytes $(12,14)$, and subsequently induces astrocyte dysfunction $(2,12)$ by binding to transforming growth factor $\beta$ receptor 2 . This induces the transforming cascade of astrocytes $(13,15)$ and downregulates the inward-rectifying potassium channels in astrocytes, which may ultimately lead to N-methyl-D-aspartate-receptor-mediated neuronal hyperexcitability or a seizure (13). To date, studies in this field have predominantly focused on the effects of astrocyte albumin absorption. However, we hypothesize that, after infiltrating into the brain parenchyma, serum albumin is not only absorbed by astrocytes (16) but also by neurons; therefore, the effects of neuronal albumin uptake were investigated in the present study.

Temporal lobe epilepsy (TLE) is one of the most common types of epilepsy with significant BBB damage and albumin extravasation (17). In the present study, a rat model of TLE 
was established in order to simulate BBB damage and explore the effect of serum albumin extravasation during an epileptic seizure. This may aid in the search of a novel target for epilepsy treatment.

\section{Materials and methods}

Ethical approval. The present study was approved by the Ethic Committee of Shanghai Tongji University (Shanghai, China). All experimental procedures were conducted in strict accordance with the standards outlined by the Association for Assessment and Accreditation of Laboratory Animal Care (18). All efforts were made to minimize the number of animals involved.

Animal preparation. A total of 60 male ( $n=10$ per group) Sprague-Dawley rats, weighing 250-300 g, were purchased from Shanghai Super-B\&K Laboratory Animal Corp. Ltd (Shanghai, China) and maintained in temperature $\left(21 \pm 2^{\circ} \mathrm{C}\right)$ and humidity $(60 \%)$ controlled environment. Mice used for modeling were 8-9 weeks old, whereas 18 day-old mice were used for neuron culture. Prior to and following surgery, mice were maintained in plastic cages with a $12 \mathrm{~h}$ light/dark cycle and ad libitum access to food and water.

Kainic acid (KA) injection and electrode implantation. Following anesthetization with $0.4 \mathrm{~g} / \mathrm{kg}$ pentobarbital sodium (2\%) (Sigma-Aldrich, St. Louis, MO, USA), rats were fixed on stereotaxic apparatus. During surgery, the fonticulus anterior, sagittal suture and fonticulus posterior were adequately exposed and adjusted into the same horizontal plane. A microchannel was drilled (bregma: anteroposterior, $-3.6 \mathrm{~mm}$; mediolateral, -3.4 mm; dorsoventral, -3.8 mm) for intrahippocampal administration of KA. Preliminary testing was conducted to explore the optimal dosage of $\mathrm{KA}$, and $1 \mu \mathrm{KA}$ $(0.5 \mathrm{mM} ; 0.05 \mu \mathrm{l} / \mathrm{min}$ ) was chosen. Rats in the control group were injected with $1 \mu$ l sterile saline instead of KA. Deep recording electrodes (Electroencephalograph Analysis System version 1.70; Cadwell Laboratories, Inc., Kennewick, WA, USA) were bilaterally implanted into the hippocampal CA3 region using the same coordinates. A monopolar reference electrode was implanted into the bone above the cerebellum. All electrodes were fixed to the skull using screws and embedded by dental cement (Paladur; Heraeus Kulzer, Ltd., Newbury, UK). Following the surgery, both electroencephalogram (EEG) recording and behavioral monitoring were conducted for $12 \mathrm{~h}$. Behavioral characteristics were evaluated according to Racine's 5-point scale: 0, motionless, excitement and rapid breathing; 1 , movement of the mouth, lips, tongue and vibrissae; 2 , head clonus; 3 , forelimb clonus with lordotic posture and 'wet dog shakes'; 4, forelimb clonus, with rearing and falling; and 5, generalized tonic-clonic activity with loss of postural tone, often in death, and wild jumping (19).

Sampling. Following anesthetization with $10 \%$ pentobarbital sodium, rats were sacrificed via cardiac-perfusion with $70 \mathrm{ml}$ warmed saline and $40 \mathrm{ml} 4 \%$ paraformaldehyde (PFA; $4^{\circ} \mathrm{C}$; PH 7.4; Sigma-Aldrich). Intact brains were immediately excised, immersed in 4\% PFA for $18 \mathrm{~h}$, and subsequently dehydrated with $30 \%$ sucrose (in $0.9 \%$ normal saline; PH 7.4 ) for 3 days at $4{ }^{\circ} \mathrm{C}$. To ensure the dehydration efficiency, the $30 \%$ sucrose was changed daily. Following this, the samples were dried, embedded in Optimum Cutting Temperature compound (Shandon Cryomatrx embedded resin; Thermo Fisher Scientific, Inc., Waltham, MA, USA) and stored at $-80^{\circ}$ prior to immunoassay analysis.

Nissl staining for neuronal injury. Nissl staining was performed to detect neuronal injury. Sections were cut to 35- $\mu \mathrm{M}$ thickness using a sliding microtome (CM1950; Leica Microsystems GmbH, Wetzlar, Germany). Following washing with phosphate-buffered saline (PBS; $\mathrm{pH} 7.4$ ), tissue sections were dried at $55^{\circ} \mathrm{C}$ for $3 \mathrm{~h}$, immersed in $0.9 \%$ cresyl violet (860980; Sigma-Aldrich) for $2 \mathrm{~h}$, dehydrated with 70, 80, 90 and $100 \%$ ethanol, respectively, for $5 \mathrm{~min}$ each and finally mounted with neutral balsam (Beijing Solarbio Science \& Technology Co., Ltd., Beijing, China). Stained tissue sections were observed using a Nikon Eclipse 80i optical microscope (Nikon Corporation, Tokyo, Japan).

Primaryculture ofhippocampalneurons andalbuminabsorption. Hippocampal neurons were dissected from rat embryos (18th day of gestation; Shanghai Super-B\&K Laboratory Animal Corporation, Ltd., Shanghai, China) via treatment with $10 \mathrm{U} / 1$ papain solution (Sigma-Aldrich). Primary neurons $\left(2 \times 10^{5} / \mathrm{ml}\right)$ were seeded on poly-L-lysine-coated dishes and cultured in minimum essential medium supplemented with $5 \%$ fetal bovine serum (both purchased from Signa-Aldrich) and $5 \%$ horse serum (Thermo Fisher Scientific, Inc.) under $5 \% \mathrm{CO}_{2}$ conditions on the first day. On the second day, cells were cultured in neurobasal medium with B27 (Thermo Fisher Scientific, Inc.), containing $0.025 \mathrm{mM}$ glutamate; whereas on the third day, cells were treated with $5 \mu \mathrm{M}$ cytosine-1- $\beta$-D-arabinofuranoside (Sigma-Aldrich)to inhibit the growth of gliocytes. After two days, half of the neurobasal medium was replaced by fresh medium. Cells were subsequently treated with saline, $10 \mu \mathrm{M} \mathrm{KA}$, $10 \mu \mathrm{M} \mathrm{KA}+10 \mu \mathrm{M}$ albumin, $10 \mu \mathrm{M} \mathrm{KA}+50 \mu \mathrm{M}$ albumin and $10 \mu \mathrm{M} \mathrm{KA}+100 \mu \mathrm{M}$ albumin at $37^{\circ} \mathrm{C}$ and $5 \% \mathrm{CO}_{2}$ for $24 \mathrm{~h}$. Staining of Evans blue (EB) and immunofluorescent staining of NeuN were performed after cell were washed with PBS.

Double immunofluorescence. For double immunofluorescence, rats were injected with $4 \mathrm{ml} / \mathrm{kg} \mathrm{EB} \mathrm{(2 \% ),} \mathrm{which} \mathrm{is}$ capable of directly binding to albumin, via the tail vein $4 \mathrm{~h}$ prior to sacrifice via trans-cardiac perfusion. EB administration was performed on days $0,3,5,7,14,21$ and 28 following $\mathrm{KA}$ injection. Cell slices were also stained with EB in the dark overnight. Brain $(35 \mu \mathrm{M})$ and cell sections were subsequently washed in cold PBS and incubated with monoclonal primary mouse anti-rat anti-NEUN (dilution, 1:1,000; A2050; Sigma-Aldrich) at $4^{\circ} \mathrm{C}$ overnight. Following washing three times with PBS for $15 \mathrm{~min}$, sections were incubated with polyclonal AF488-conjugated goat anti-mouse secondary antibody (dilution, 1:2,000; A-11001; Invitrogen; Thermo Fisher Scientific, Inc.) for $3 \mathrm{~h}$ at room temperature. Sections were three times washed with PBS for $5 \mathrm{~min}$ and mounted with $75 \%$ glycerin (Sigma-Aldrich). Images were captured using a Nikon Eclipse 80i luminescence microscope. 
Table I. Baseline analysis.

\begin{tabular}{lccc}
\hline Group & Weight $(\mathrm{g})$ & Grade of seizure & Mortality rate \\
\hline Control group & $286.03 \pm 2.51$ & $0.00 \pm 0.00$ & 0 \\
Saline injection group & $282.94 \pm 2.93$ & $0.00 \pm 0.00$ & 0 \\
Kainic acid group & $284.21 \pm 2.71$ & $4.88 \pm 0.32$ & 2 \\
\hline
\end{tabular}

No significant differences in weight were detected at the baseline level among the groups.

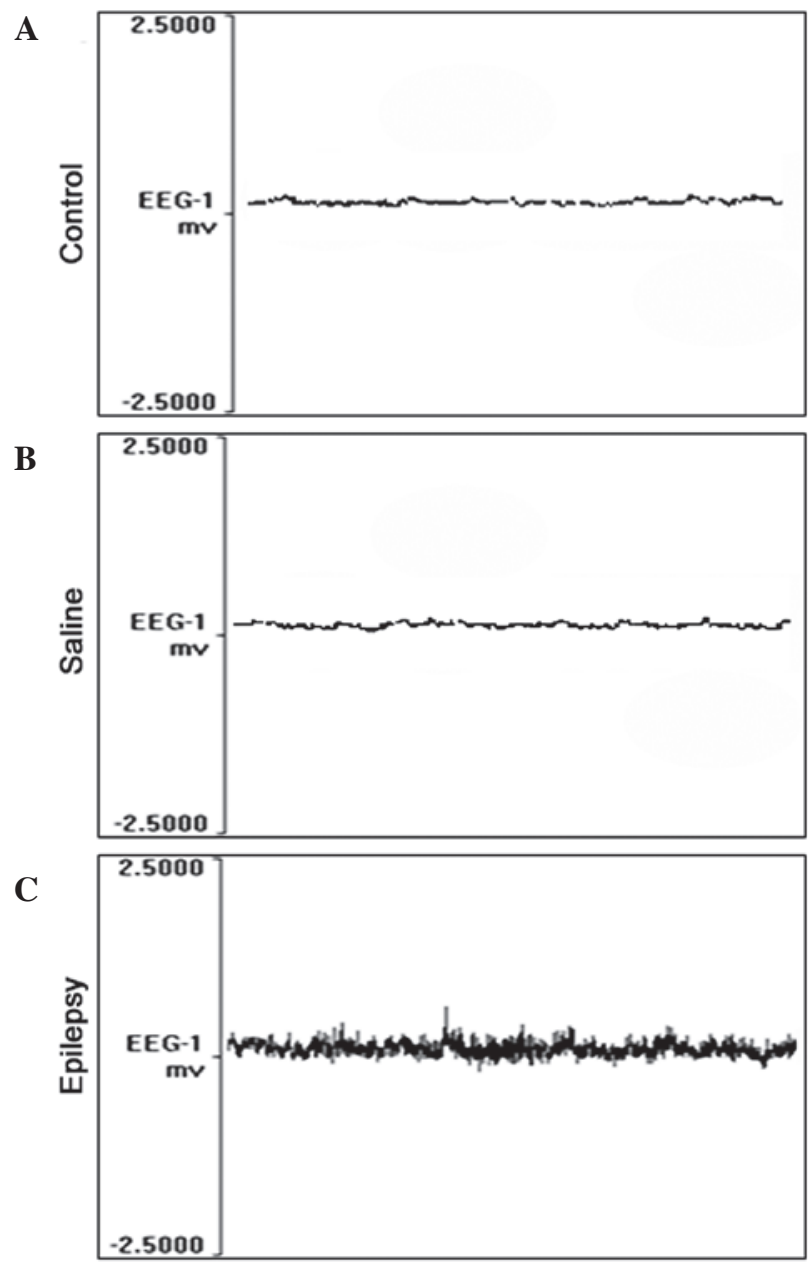

Figure 1. Electroencephalogram (EEG) recording. Rats in the (A) control and (B) saline groups exhibited normal brain activity, whereas large spikes were detected in the $(\mathrm{C})$ temporal lobe epilepsy group.

Overlap coefficient analysis. To explore the relationship between neurons and albumin extravasation, overlap coefficient analysis was performed to analyze the overlap degree of the two fluorescence signals (0.0-1.0). Analyses were conducted using Image-Pro Plus 6.0 (Media Cybernetics, Inc., Rockville, MD, USA) in all rats and cell slices, and two-dimensional curve graphs, with fluorescence intensity on the vertical axis and regional range on the horizontal axis, of the two fluorescence signals were calculated from the entire image.

Statistical analysis. All data were presented as the mean \pm standard error of the mean following analysis by SPSS 16.0 (SPSS, Inc., Chicago, IL, USA). Inter-group comparisons were analyzed using analysis of variance. Analysis of the overlap coefficient between different cells was conducted by Image-Pro Plus 6.0.

\section{Results}

Baseline and behavior analysis. No significant differences in body weight were detected among the rats in the different groups (Table I). Behavior analysis demonstrated that stereotactic injection of KA induced grade 4-5 seizure attacks (Table I). Two rats succumbed to seizure attacks during EEG recording; these two rats were excluded from subsequent analyses.

EEG recording. Rats in the control (Fig. 1A) and saline groups (Fig. 1B) exhibited normal brain activities, whereas large spikes were observed following KA injection (Fig. 1C) in the rat model of TLE. These results demonstrated that a rat model of TLE was successfully established.

Neuronal albumin absorption mirrors neuronal damage in vivo and in vitro. Nissl staining demonstrated normal distribution and characteristics of neurons in the hippocampi and the surrounding area (Fig. 2A) in the control group. Conversely, marked neuronal damage was detected in the KA-induced TLE model group. Neuronal damage was predominantly distributed in the hippocampus (CA1, CA2, $\mathrm{CA} 3$, and dentate gyrus) surrounding the injection site of KA (Figs. 2B and C). As demonstrated by EB staining, no albumin extravasation was detected in the control group (Fig. 2D); whereas, the distribution of albumin extravasation in the TLE model groups (Figs. 2E and F) was consistent with the neuronal damage detected by Nissl staining. Furthermore, EB staining on the 14th day of KA demonstrated severe atrophy of the hippocampus with significant loss of lesion volume $(\mathrm{P}<0.001$; Fig. $2 \mathrm{H})$ and a scarcity of albumin-positive cells (Fig. 2F). Notably, minimal albumin-positive neurons were detected in the atrophied hippocampus on the 14th (Fig. 2F) and 21th day (Fig. 2G) after KA injection. Quantitative analysis of the hippocampal volume over time demonstrated a similar result. Hippocampal volume significantly decreased on days $14(\mathrm{P}<0.001)$ and $21(\mathrm{P}=0.12)$ post-seizure, respectively, in the KA-induced TLE group, as compared with the control group (Fig. 2H). These results indicate that neuronal albumin update is a hallmark of neuronal damage.

Serum albumin is significantly absorbed by neurons. Albumin extravasation was not observed in the control group (Fig. 2D), 

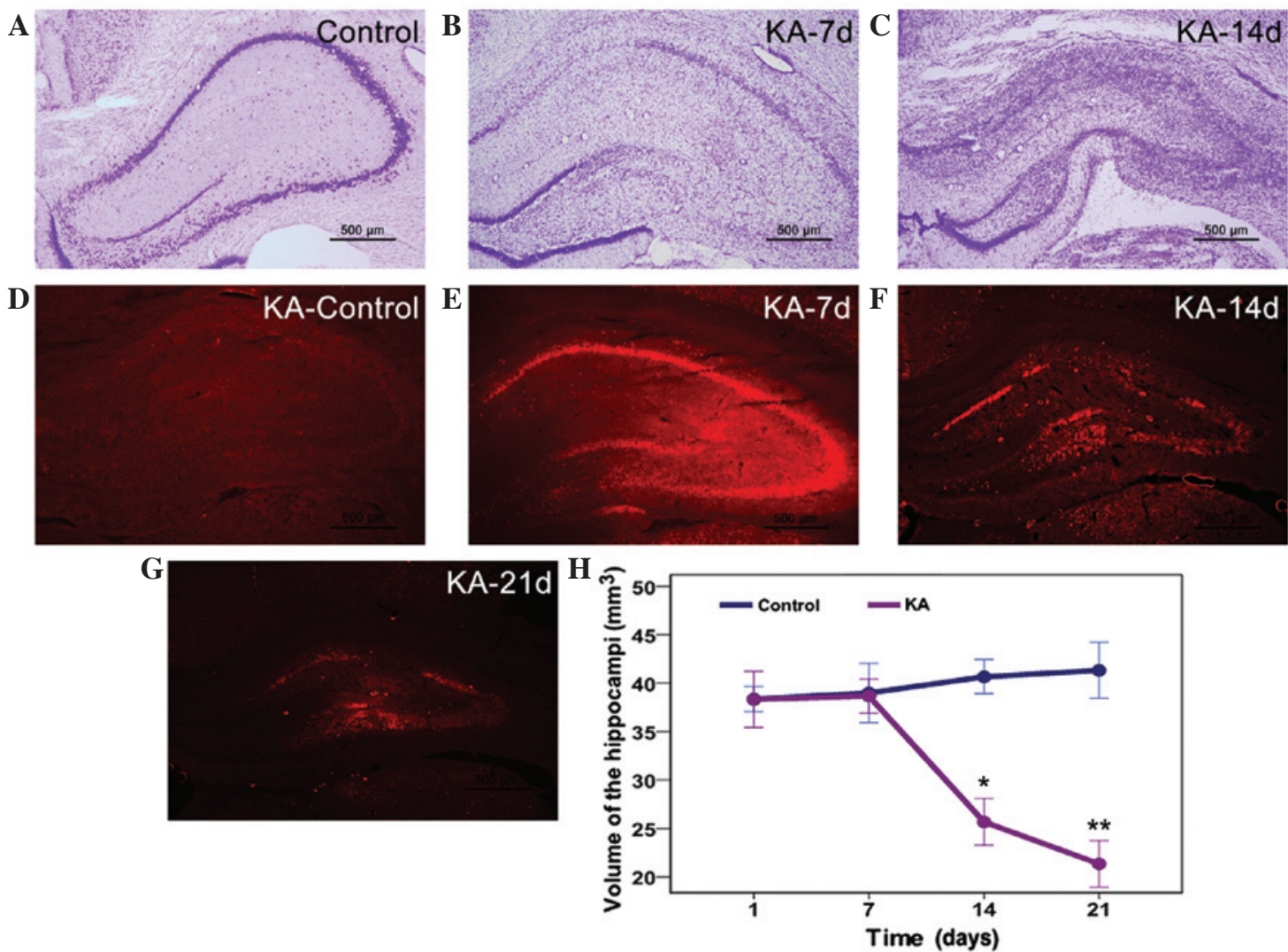

Figure 2. Neuronal albumin uptake correlates with neuronal injury, as detected by (A-C) Nissl staining and (D-G) Evans blue staining. Rats in the control group exhibited (A) normal hippocampal structure and (D) non-significant albumin leakage. (B) On the 7th day post-KA injection, Nissl staining demonstrated significant neuronal damage in the hippocampus around the KA injection site, (E) which was consistent with the distribution of albumin extravasation on the 7 th day in the KA group. On the (C and F) 14th and $(\mathrm{G}) 21$ st days following KA injection, the hippocampi of rats in the KA group exhibited severe atrophy with significant loss of lesion volume and (F) albumin-positive cells, as compared with the (D) control and (E) KA-7 day group. (F and G) Minimal albumin-positive neurons were detected in the atrophied hippocampi. (H) Quantitative analysis showed a similar result. Scale bar, $500 \mu \mathrm{m}$. ${ }^{*} \mathrm{P}<0.001$ and ${ }^{* *} \mathrm{P}=0.12$ vs. the control group. KA, kainic acid.

whereas EB staining in KA group indicated widespread albumin leakage (Figs. 2E-G). As a result, the distribution of extravasated albumin (Fig. 3A) was similar to the distribution of NeuN neurons (Fig. 3B). Merging of EB and NeuN staining showed co-localization of albumin and neurons following KA injection (Fig. 3C). Compared with the control group (Fig. 3D), KA injection resulted in the down-regulation of NeuN (Fig. 3E), indicating that these neurons were injured by KA. The merging (Fig. 3H) of amplified images of albumin (Fig. 3F) and NeuN (Fig. 3G) staining further showed similar morphological characteristics and co-localization of albumin and neurons. Overlap analysis showed the similar intensity of albumin and neurons (Fig. 3I) in a different position. These findings are consistent with the results of double immunofluorescence in vitro, suggesting that serum albumin is absorbed by neurons after extravasation.

Albumin absorption aggravates neuronal damage in vitro. To further explore whether albumin extravasation promoted neuron damage, in vitro experiments were performed. The control group showed normal numbers and morphological characteristics of neurons in vitro (Fig. 4A). Following KA treatment (Fig. 4B), the number of neurons was significantly reduced compared with the control group $(\mathrm{P}<0.01)$. The $\mathrm{KA}+10 \mu \mathrm{M}$ and $\mathrm{KA}+50 \mu \mathrm{M}$ albumin groups showed significantly aggravated neuronal damage and death in a dose-dependent manner $(\mathrm{P}<0.05)$ (Fig. 4C and D). Staining of NeuN (Fig. 4E) and EB (Fig. 4F) in vitro showed co-localization and similar morphological characteristics of albumin and neurons (Fig. 4G), consistent with the result in vivo. Cell counting showed that the dose tie of $10 \mu \mathrm{M}$ albumin contributed to more neuronal death at $48 \mathrm{~h}$ compared with at $24 \mathrm{~h}$ $(\mathrm{P}<0.05$; Fig. $4 \mathrm{H})$. These results indicate that albumin uptake aggravates neuronal damage following injury by KA.

\section{Discussion}

With the progression of experimental and clinical research, albumin leakage has been associated with various central nervous diseases including epileptic seizure (2), gangliogliomas (8) and Alzheimer's disease (20). Therefore, it is crucial that albumin extravasation and its underlying effects are investigated and understood. Previous studies have indicated that albumin leakage occurs due to BBB damage induced by epileptic seizures $(12,13)$. Furthermore, it has been demonstrated that BBB dysfunction and subsequent serum albumin extravasation contribute to epileptogenesis and the pathology of idiopathic and symptomatic epilepsy $(5,21)$. The present study used a KA-induced rat model of TLE to explore the association between extravasated albumin and neurons 

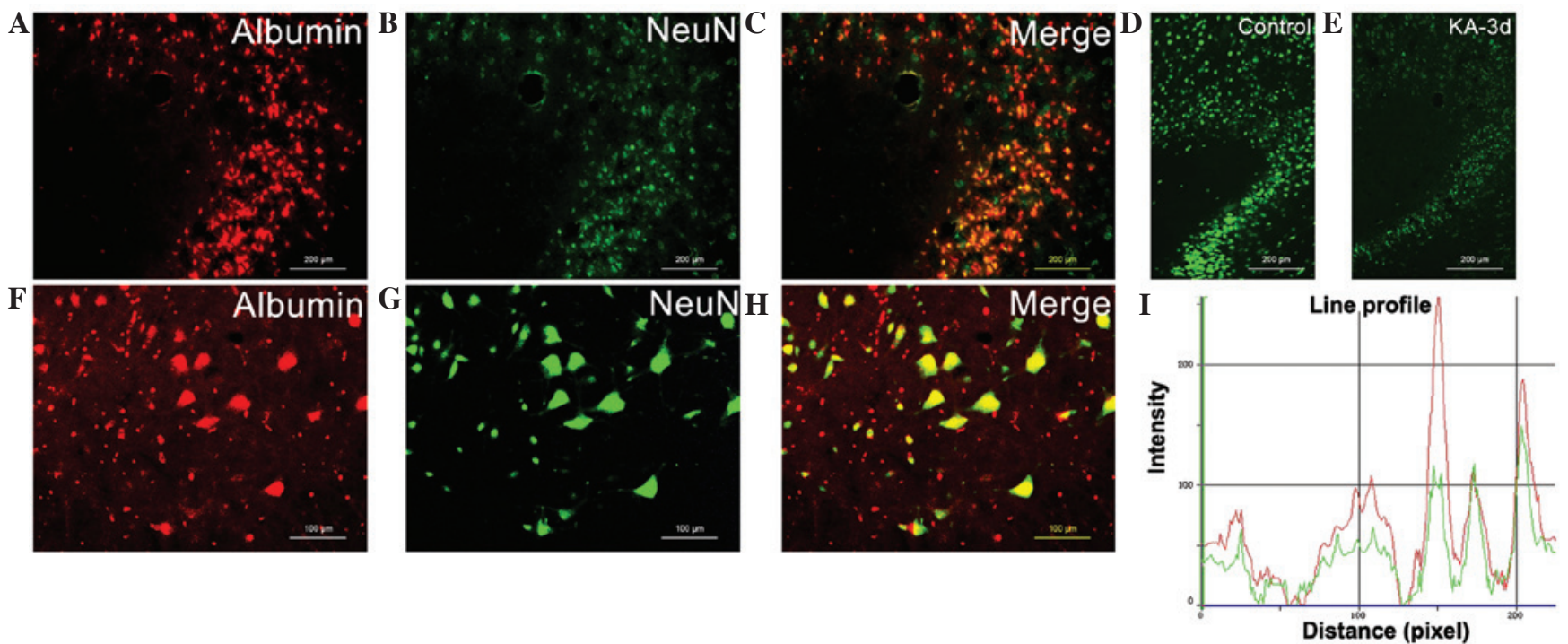

Figure 3. Serum albumin was significantly absorbed by neurons. (A and F) Evans blue staining of kainic acid showed the extravasation of albumin. (B and G) NeuN staining of neurons. (C and H) Merged images of Evans blue and NeuN staining. NeuN expression levels were reduced in neurons following injection with (E) KA, compared with the (D) control group. (I) Line profile graph results showed an association between the immune-intensity of albumin (red) and NeuN (green) in different positions. Scale bar: (A-E), $200 \mu \mathrm{m}$; (F-I), $50 \mu \mathrm{m}$. KA, kainic acid.

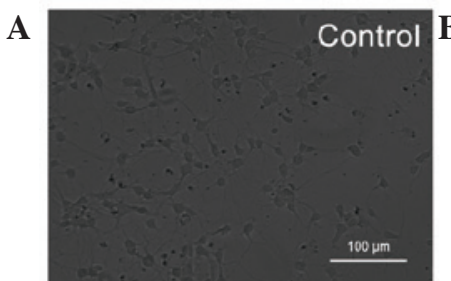

$\mathbf{E}$

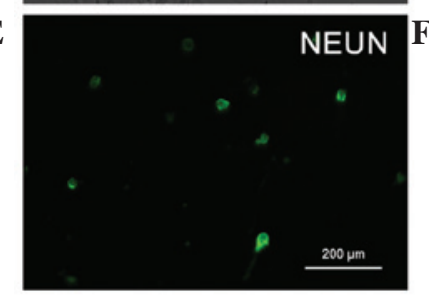

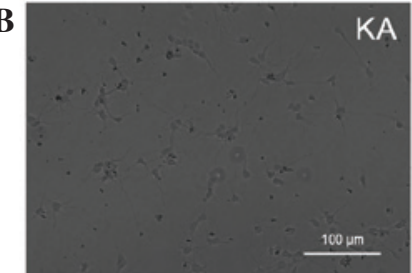
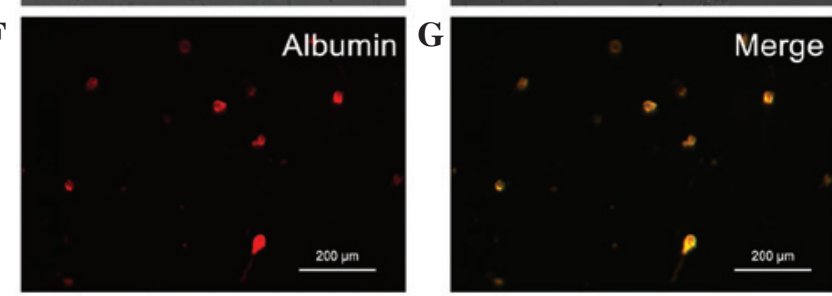

$\mathbf{H}$

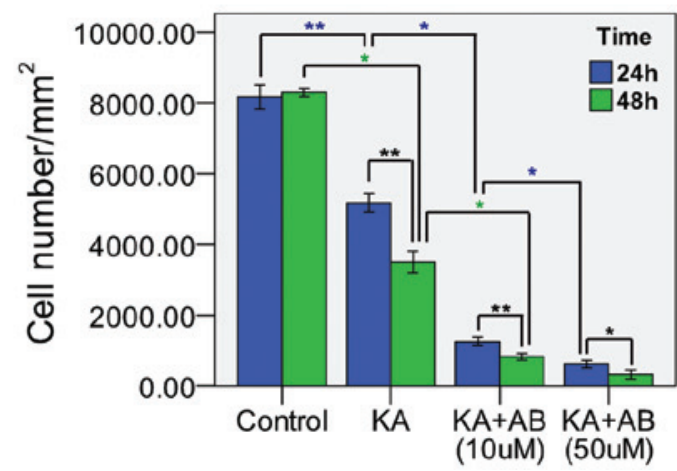

Figure 4. Albumin absorption aggravated neuronal damage in vitro. As compared with the (A) control group, (B) KA (10 uM) treatment in vitro induced significant neuronal damage. As compared with these groups, (C) KA + albumin (10 uM) and (D) KA + albumin (50 uM) treatment induced more severe neuronal damage and neuronal death in a dose- and time-dependent manner. Double staining of (E) NeuN and (F) albumin demonstrated a match between NeuN and albumin in vitro. $(\mathrm{H})$ Cell counting showed reduced neuron number of albumin treatment in a dose- and time-dependent manner. ${ }^{*} \mathrm{P}<0.05,{ }^{* *} \mathrm{P}<0.01$. Scale bar: (A-D), $100 \mu \mathrm{m}$; (E-G), $200 \mu \mathrm{m}$. KA, kainic acid.

in vitro and in vivo in order to assess the effect of albumin extravasation.

Although albumin uptake by astrocytes is well documented $(12,22)$, the result of albumin extravasation has not been well explored. The present study demonstrated marked albumin leakage in a KA-induced rat model of TLE on days 7-21 after a seizure attack. Complete overlap was demonstrated between albumin- and NEUN-positive neurons, as 
detected by double immunofluorescence in vitro and in vivo. Furthermore, the results of overlap coefficient analysis were consistent with this phenomenon. These results supported the hypothesis that albumin is significantly absorbed by neurons, which is consistent with a previous study (23).

Vega et al (24) have previously demonstrated that the presence of albumin protects neurons by promoting neuronal survival, maintaining neuronal differentiation, resisting oxidation and diminishing hypoxia injury $(24,25)$. However, a recent study indicated that albumin distribution in the wrong region of the brain, such as in the hippocampus, correlates with neuronal hyperexcitability and epileptiform activities (2). Therefore, whether albumin extravasation into the brain is beneficial or detrimental remains controversial $(26,27)$. In the present study, the distribution of injured neurons and albumin extravasation was co-localized in rat models of TLE. Furthermore, on the 14th and 21st day post-seizure, hippocampi with albumin extravasation exhibited severe atrophy with significant loss of lesion volume and albumin-positive neurons. As compared with the contralateral hippocampus in the rat brain of the KA group, minimal albumin-positive neurons remained in the atrophied hippocampus, indicating that the majority of albumin-positive neurons had died and dissolved. To further explore the correlation between albumin absorption and neuronal death, in vitro studies were also performed. The results indicated that albumin treatment significantly aggravated neuronal damage and death in a doseand time-dependent manner. Double staining in vitro also demonstrated a co-localization between albumin and damaged neurons. These results suggested that albumin may be toxic to neurons and may have a key role in the promotion of neuronal cell death in rat models of TLE.

In conclusion, the results of the present study indicated that albumin was significantly absorbed by neurons and suggested that neuronal uptake of albumin may be associated with neuron damage and death during epileptic seizure. Therefore, whether attenuating albumin extravasation following epileptic seizures is capable of reducing brain damage and slowing epilepsy development requires further study. Furthermore, although the results of the study suggested that large amounts of albumin uptake may be associated with neuronal injury, we hypothesize that there may be a threshold determining whether albumin uptake is toxic or not. Further study is required to investigate this hypothesis.

\section{Acknowledgements}

The present study was supported by a grant from the National Natural Science Foundation of China (grant no. 81271441). The authors would like to thank Professor YuQiang Ding and his colleagues (affiliated to Tongii University, Shanghai, China) for the technical and theoretical support.

\section{References}

1. Inci A, Gencpinar P, Orhan D, Uzun G, Ozdem S, Samur AA, Haspolat $\mathrm{S}$ and Duman O: Ischemia-modified albumin levels in children having seizure. Brain Dev 35: 849-852, 2013.

2. Braganza O, Bedner P, Hüttmann K, von Staden E, Friedman A, Seifert G and Steinhäuser C: Albumin is taken up by hippocampal NG2 cells and astrocytes and decreases gap junction coupling. Epilepsia 53: 1898-1906, 2012.
3. Suidan GL, McDole JR, Chen Y, Pirko I and Johnson AJ: Induction of blood brain barrier tight junction protein alterations by CD8 T cells. PLoS One 3: e3037, 2008.

4. Roberts J, Kahle MP and Bix GJ: Perlecan and the blood-brain barrier: Beneficial proteolysis? Front Pharmacol 3: 155, 2012.

5. Librizzi L1, Noè F, Vezzani A, de Curtis M and Ravizza T: Seizure-induced brain-borne inflammation sustains seizure recurrence and blood-brain barrier damage. Ann Neurol 72: 82-90, 2012.

6. Herrera-Peco I, Sola RG, Osejo V, Wix-Ramos R and Pastor J: Role of astrocytes activated by albumin in epileptogenesis. Rev Neurol 47: 582-587, 2008

7. Weissberg I, Reichert A, Heinemann U and Friedman A: Blood-brain barrier dysfunction in epileptogenesis of the temporal lobe. Epilepsy Res Treat 2011: 143908, 2011.

8. Lapilover EG, Lippmann K, Salar S, Maslarova A, Dreier JP, Heinemann U and Friedman A: Peri-infarct blood-brain barrier dysfunction facilitates induction of spreading depolarization associated with epileptiform discharges. Neurobiol Dis 48: 495-506, 2012.

9. Ivens S, Gabriel S, Greenberg G, Friedman A and Shelef I: Blood-brain barrier breakdown as a novel mechanism underlying cerebral hyperperfusion syndrome. J Neurol 257: 615-620, 2010.

10. Defazio RA, Zhao W, Deng X, Obenaus A and Ginsberg MD: Albumin therapy enhances collateral perfusion after laser-induced middle cerebral artery branch occlusion: a laser speckle contrast flow study. J Cereb Blood Flow Metab 32: 2012-2022, 2012.

11. Is M, Uzan M, Unal F, Kiris T, Tanriverdi T, Mengi $M$ and Kilic N: Intraventricular albumin: An optional agent in experimental post-traumatic brain edema. Neurol Res 27: 67-72, 2005

12. Ralay Ranaivo H, Hodge JN, Choi N and Wainwright MS: Albumin induces upregulation of matrix metalloproteinase-9 in astrocytes via MAPK and reactive oxygen species-dependent pathways. J Neuroinflammation 9: 68, 2012.

13. Ivens S, Kaufer D, Flores LP, Bechmann I, Zumsteg D, Tomkins O, Seiffert E, Heinemann U and Friedman A: TGF-beta receptor-mediated albumin uptake into astrocytes is involved in neocortical epileptogenesis. Brain 130: 535-547, 2007.

14. Schmitz AK, Grote A, Raabe A, Urbach H, Friedman A, von Lehe M, Becker AJ and Niehusmann P: Albumin storage in neoplastic astroglial elements of gangliogliomas. Seizure 22: 144-150, 2013.

15. Cacheaux LP, Ivens S, David Y, Lakhter AJ, Bar-Klein G, Shapira M, Heinemann U, Friedman A and Kaufer D: Transcriptome profiling reveals TGF-beta signaling involvement in epileptogenesis. J Neurosci 29: 8927-8935, 2009.

16. Iffland PH II, Carvalho-Tavares J, Trigunaite A, Man S, Rasmussen P, Alexopoulos A, Ghosh C, Jørgensen TN and Janigro D: Intracellular and circulating neuronal antinuclear antibodies in human epilepsy. Neurobiol Dis 59: 206-219, 2013.

17. Tellez-Zenteno JF and Ladino LD: Temporal epilepsy: Clinical, diagnostic and therapeutic aspects. Rev Neurol 56: 229-242, 2013 (In Spanish).

18. Association for Assessment and Accreditation of Laboratory Animal Care International: AAALAC International rules of accreditation. http://www.aaalac.org/. Accessed January, 2012.

19. Lüttjohann A1, Fabene PF and van Luijtelaar G: A revised Racine's scale for PTZ-induced seizures in rats. Physiol Behav 98: 579-586, 2009.

20. Algamal M, Milojevic J, Jafari N, Zhang W and Melacini G: Mapping the interactions between the Alzheimer's A $\beta$-peptide and human serum albumin beyond domain resolution. Biophys J 105: 1700-1709, 2013

21. Frigerio F, Frasca A, Weissberg I, Parrella S, Friedman A, Vezzani A and Noé FM: Long-lasting pro-ictogenic effects induced in vivo by rat brain exposure to serum albumin in the absence of concomitant pathology. Epilepsia 53: 1887-1897, 2012.

22. Raabe A, Schmitz AK, Pernhorst K, Grote A, von der Brelie C, Urbach H, Friedman A, Becker AJ, Elger CE and Niehusmann P: Cliniconeuropathologic correlations show astroglial albumin storage as a common factor in epileptogenic vascular lesions. Epilepsia 53: 539-548, 2012.

23. Sharma HS, Miclescu A and Wiklund L: Cardiac arrest-induced regional blood-brain barrier breakdown, edema formation and brain pathology: A light and electron microscopic study on a new model for neurodegeneration and neuroprotection in porcine brain. J Neural Transm 118: 87-114, 2011. 
24. Vega L, Arroyo AA, Tabernero A and Medina JM: Albumin-blunted deleterious effect of amyloid-beta by preventing the internalization of the peptide into neurons. J Alzheimers Dis 17: 795-805, 2009.

25. Sutherland BA, Minnerup J, Balami JS, Arba F, Buchan AM and Kleinschnitz C: Neuroprotection for ischaemic stroke: Translation from the bench to the bedside. Int J Stroke 7: 407-418, 2012.
26. Bath PM: Albumin for hyperacute stroke: Another failed neuroprotectant. Lancet Neurol 12: 1036-1037, 2013.

27. Sharma HS, Miclescu A and Wiklund L: Cardiac arrest-induced regional blood-brain barrier breakdown, edema formation and brain pathology: A light and electron microscopic study on a new model for neurodegeneration and neuroprotection in porcine brain. J Neural Transm 118: 87-114, 2011. 